\title{
Effect of Olive Mill Wastewaters on Scenedesmus Sp. Growth, Metabolism and Phenols Removal
}

\section{Ines Dahmen-Ben Moussa ( $\nabla$ ines_dahmen@hotmail.fr )}

CBS https://orcid.org/0000-0001-7121-0150

\section{Mohamed Ali Masmoudi}

Centre de Biotechnologie de Sfax

\section{Firas Feki}

Centre de Biotechnologie de Sfax

\section{Sirine Choura}

Centre de Biotechnologie de Sfax

\section{Sami Sayadi}

Center for Sustainable development college of arts and sciences, Qatar

\section{Research}

Keywords: Scenedesmus sp., olive mill wastewater, antioxidant molecules, Lipid, phenol removal

Posted Date: May 29th, 2020

DOI: https://doi.org/10.21203/rs.3.rs-28352/v2

License: (c) (1) This work is licensed under a Creative Commons Attribution 4.0 International License. Read Full License 
The authors have withdrawn this preprint from Research Square 